\title{
Electron Mirror-mode Structure: Magnetospheric Multiscale Observations
}

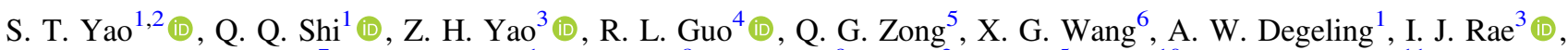

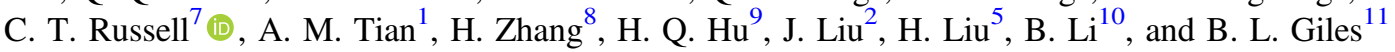 \\ ${ }^{1}$ Shandong Provincial Key Laboratory of Optical Astronomy and Solar-Terrestrial Environment, Institute of Space Sciences, Shandong University, Weihai, 264209, \\ People's Republic of China; sqq@sdu.edu.cn \\ ${ }^{2}$ State Key Laboratory of Space Weather, National Space Science Center, Chinese Academy of Sciences, Beijing 100190, People's Republic of China \\ ${ }^{3}$ Laboratoire de Physique Atmosphérique et Planétaire, STAR Institute, Université de Liège, Liège, Belgium \\ ${ }^{4}$ Key Laboratory of Earth and Planetary Physics, Institute of Geology and Geophysics, Chinese Academy of Sciences, Beijing 100029, People's Republic of China \\ ${ }^{5}$ School of Earth and Space Sciences, Peking University, Beijing, 100871, People's Republic of China \\ ${ }_{7}^{6}$ Department of Physics, Harbin Institute of Technology, Harbin 150001, People's Republic of China \\ ${ }^{7}$ Department of Earth, Planetary and Space Sciences, University of California, Los Angeles, CA, USA \\ ${ }^{8}$ Physics Department and Geophysical Institute, University of Alaska Fairbanks, Fairbanks, AK, USA \\ ${ }^{9}$ SOA Key Laboratory for Polar Science, Polar Research Institute of China, Shanghai, People's Republic of China \\ ${ }^{10}$ Chinese Antarctic Center of Surveying and Mapping, Wuhan University, Wuhan, 430079, People's Republic of China \\ ${ }^{11}$ NASA Goddard Space Flight Center, Greenbelt, MD, USA \\ Received 2019 July 3; revised 2019 July 16; accepted 2019 July 16; published 2019 August 19
}

\begin{abstract}
The small-scale mirror mode excited by electron dynamics is a fundamental physical process, attracting research interest in space, laboratory, and astrophysical plasma physics over the past half century. However, the investigations of this process were mostly limited to theories and numerical simulations, with no direct observational evidence for their existence. In this study we present clear observations of electron mirror-mode using Magnetospheric Multiscale data at unprecedented high temporal cadence. These structures are train-like, compressible, nonpropagating, and satisfy the theoretical excitation and electron trapping conditions. They were observed near the Earth's foreshock and its downstream turbulence during the corotating interaction region events, which could be involved with the interaction between solar wind and Earth.
\end{abstract}

Unified Astronomy Thesaurus concepts: Space plasmas (1544); Planetary magnetosphere (997); Solar wind (1534); Shocks (2086)

\section{Introduction}

The mirror mode is a fundamental process in magnetized plasma environments. It plays many important roles in solar physics, interplanetary, planetary, astrophysical, and laboratory plasma environments, for example, it converts electromagnetic energy and particle kinetic energy (Kivelson \& Southwood 1996), excites plasma waves and instabilities (Smith et al. 1969), modulates particle distributions (Yao et al. 2018b), and is suggested as a significant source of turbulent energy (Pokhotelov et al. 2003) and serves as messengers of solar corona (Russell et al. 2008). The most common mirror mode is in space, for example, it is observed to be full of Earth magnetosheath and can exist in the Earth magnetosphere for a long time. These structures are generally suggested to be magnetohydrodynamics (MHD) scale and static in the plasma frame. They display anticorrelation between magnetic and plasma pressures. The spatiotemporal scales and three-dimensional (3D) structures, evolution processes, and other important effects (e.g., drift, finite Larmor radius effect, non-Maxwellian ion distribution, and electron temperature influence) have been extensively studied in the past half century (e.g., Hasegawa 1969; Southwood \& Kivelson 1993; Chisham et al. 1998; Pokhotelov et al. 2000, 2013; Gary \& Karimabadi 2006; Genot et al. 2009; Hellinger et al. 2009). The studies on the mirror mode were mostly confined to the MHD scale. Numerical simulations and theoretical studies further revealed their kinetic effects, although the fundamental theoretical framework is still at MHD scale and lacks support from observations. If the electron distribution is anisotropic (with perpendicular temperature exceeding the parallel), the electrons become unstable and would excite the electron mirror-mode instability to remove the free energy. This instability was first discovered by Basu \& Coppi (1982), and subsequently detailed by Basu \& Coppi (1984), Migliuolo (1986), Marchenko et al. (1988), and more recently by Gary \& Karimabadi (2006), Pokhotelov et al. (2013), and Hellinger \& Štverák (2018). This electron mirror-mode instability condition is similar to the MHD-scale mirror mode and can be expressed as $R_{\mathrm{e}}=\frac{T_{\mathrm{e} \perp} / T_{\mathrm{e} \|}}{1+1 / \beta_{\mathrm{e} \perp}}>1$, where $\beta_{\mathrm{e} \perp}, T_{\mathrm{e} \perp}$, and $T_{\mathrm{e} \|}$ denote electron perpendicular plasma beta and perpendicular and parallel electron temperature, respectively. This has been theoretically predicted using a linear calculation (Pokhotelov et al. 2000) and also found in more precise numerical simulations (for example, including the effect of off-diagonal terms in dielectric tensor elements for the mirror instability) by Noreen et al. (2017). The contribution of electrons in the mirror instability in the quasilinear regime was then also discussed by Noreen et al. (2017). Historically, the electron mirror mode has been studied in theories and numerical simulations. But so far there have been few unambiguous observations of their existence because the capability to resolve electron-scale structures has been strongly limited by the insufficient measurement resolution of satellite-borne instrumentation. Using magnetic field data obtained from the spacecraft AMPTE-IRM and Equator-S, Treumann \& Baumjohann (2018) presented observational evidence for the electron mirror mode in the Earth's magnetosheath. Lowfrequency whistler waves were also observed and were used to diagnose the effect of anisotropic electron temperature on the mirror mode. These observations suggest that the whistler waves were probably related to the trapped electrons for which the trapping condition was provided by the magnetic depressions caused by the electron mirror branch. The study also pointed out 

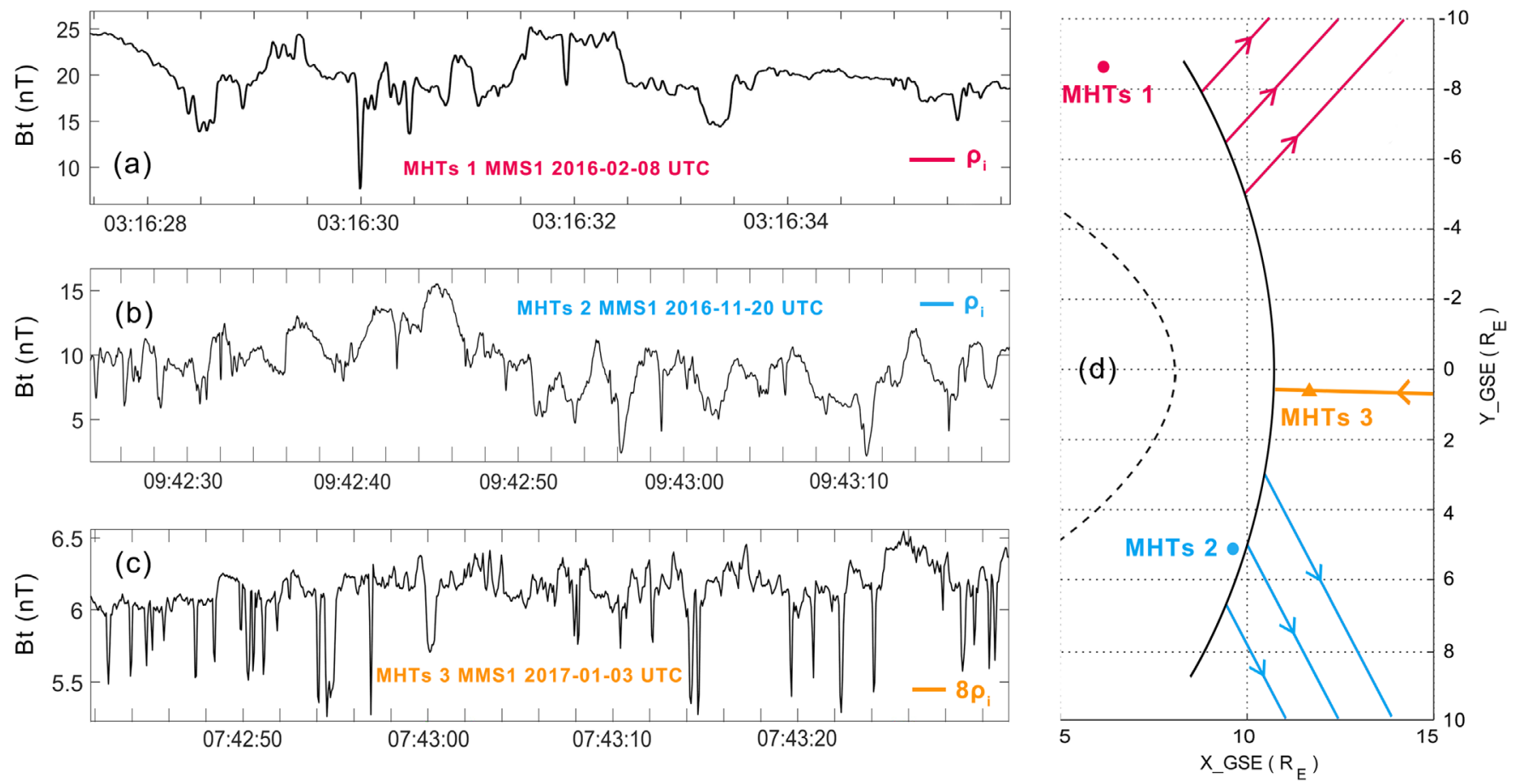

Figure 1. ((a)-(c)) Three magnetic hole trains (MHTs) observed in the solar wind plasmas. The scales of these magnetic holes were $\sim \rho_{\mathrm{i}}$. (d) The location of three MHTs in the GSE-XY plane. Arrows indicate the IMF directions of each event.

that high temporal resolution observations are required for the confirmation of the electron mirror mode and its responsibility for electron trapping and wave excitation. In the recent Magnetospheric Multiscale (MMS; Burch et al. 2016) studies, similar lowfrequency whistler waves were observed inside mirror-mode structures by Ahmadi et al. (2018) with a detailed in-depth investigation. The electron temperature anisotropy was suggested to provide a favorable environment for the growth of whistler waves. This result indicates the possible existence of localized electrons that were trapped by the electron mirror branch. Similar observations can be found in Breuillard et al. (2018), which observed whistler waves in mirror-mode structures with perpendicular electron temperature anisotropy.

One potential mirror-mode structure is the magnetic hole $(\mathrm{MH})$, a phenomenon in which the magnetic field falls and recovers rapidly within a short time, usually lasting for tens of minutes to seconds from in situ measurements, with corresponding spatial scales from thousands to tens of times the proton gyroradius $\left(\rho_{\mathrm{i}}\right)$. These have been observed in the solar wind (e.g., Winterhalter et al. 1994; Xiao et al. 2014), the magnetosphere of comets (Russell et al. 1987; Plaschke et al. 2018), the magnetospheric cusp (Shi et al. 2009), and the planetary magnetosheath (e.g., Lucek et al. 1999; Yao et al. 2018b). According to their morphological characteristics, these MHD-scale MHs can be divided into "train-like" and "isolated" categories. Train-like MHs observed downstream of the perpendicular bow shock were considered as mirror-mode structures in many studies (e.g., Lucek et al. 1999). For isolated structures, the generation mechanism remains unclear. Among the existing theories, mirror-mode and solitary wave theories are often adopted (e.g., Baumgärtel 1999; Stasiewicz 2004). In recent studies, MHs with scale sizes close to $\rho_{\mathrm{i}}$ (kinetic-scale magnetic hole, KSMH) were observed in the Earth's plasma sheet and magnetosheath (e.g., Sun et al. 2012; Sundberg et al. 2015;
Gershman et al. 2016; Goodrich et al. 2016; Yao et al. 2016; Zhang et al. 2017). The KSMH attracts attentions because it is closely related to electron-scale physical processes (e.g., Balikhin et al. 2012; Li et al. 2016; Liu et al. 2019), various kinds of plasma waves and particle acceleration (Yao et al. 2017, 2019), and energy conversion and dissipation (Huang et al. 2017; Yao et al. 2019). The KSMH displays an isolated structure whose generation mechanism is still unclear. To date, observations of train-like KSMHs have not been reported. In this study, we provide clear observations of KSMH trains in solar wind plasmas. These structures are confirmed to be electron mirror modes.

The high-resolution data from the MMS mission provide an ideal opportunity to study plasma physics at the kinetic scale. The magnetic field data taken from the Fluxgate Magnetometer instrument (Russell et al. 2016) has a sampling resolution of $16 \mathrm{~Hz}$ in survey mode and $128 \mathrm{~Hz}$ in burst mode. The Fast Plasma Investigation instrument (Pollock et al. 2016) provides measurements of electrons and ions with temporal resolution of $30 \mathrm{~ms}$ and $150 \mathrm{~ms}$, respectively. The plasma temperature, bulk velocity, and number density are calculated by taking the moments from the $3 \mathrm{D}$ particle distributions from $10 \mathrm{eV}$ to $30 \mathrm{keV}$. The solar wind conditions, including interplanetary magnetic field (IMF; resolution of $16 \mathrm{~Hz}$ ), and ion density, bulk velocity, and temperature (resolution of $64 \mathrm{~Hz}$ ) are obtained from ACE spacecraft. All the data are organized in the Geocentric Solar Ecliptic coordinates.

\section{Observations}

Figures 1(a)-(c) display three electron-scale mirror modes generated magnetic hole trains (MHTs), observed in the solar wind. The locations and the IMF directions for the three events are shown in Figure 1(d) with different colors. MHT 1 (red point) and MHT 2 (blue point) were observed downstream of the quasi-parallel shock, and MHT 3 (yellow triangle) was 

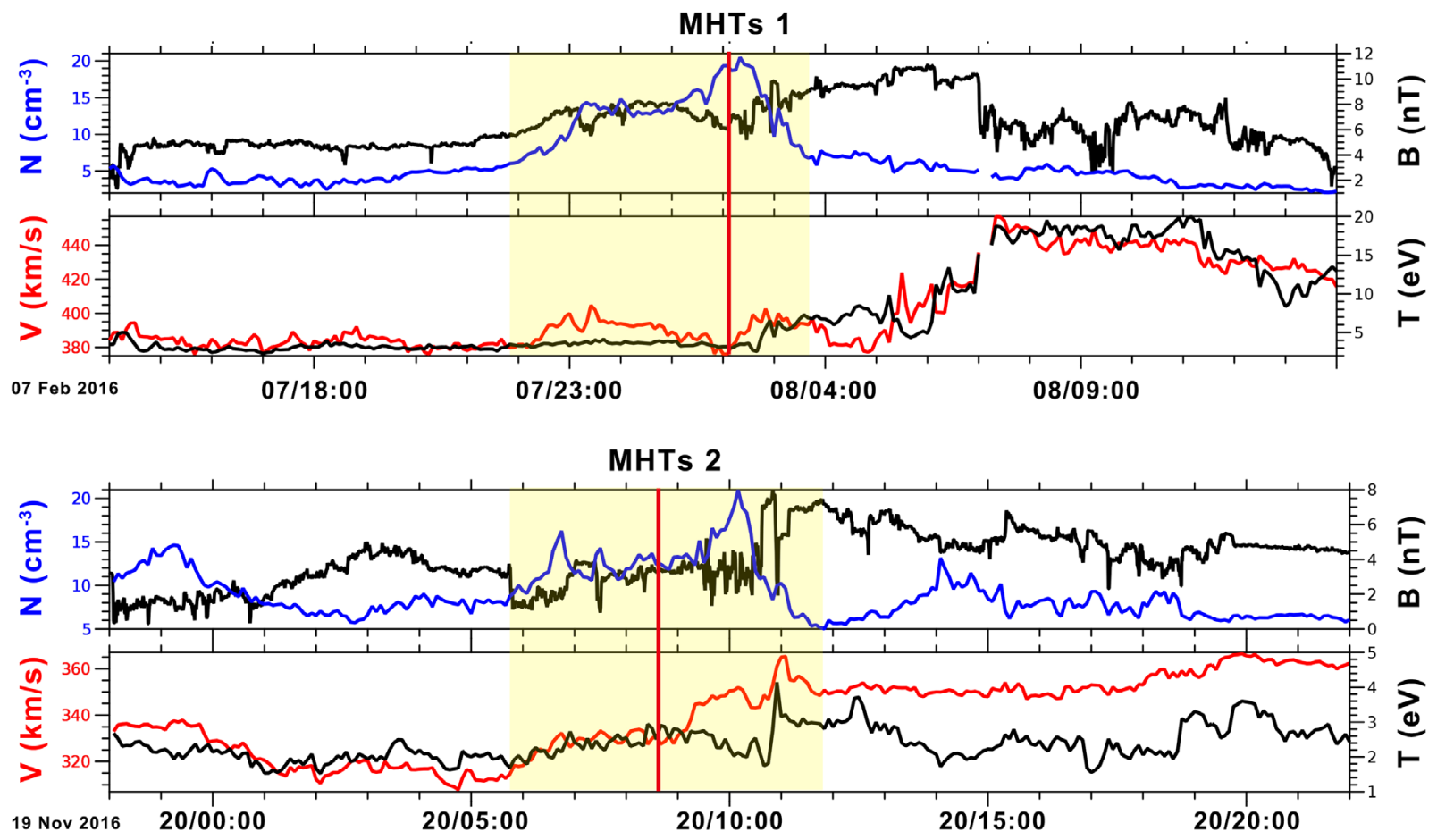

MHTs 3

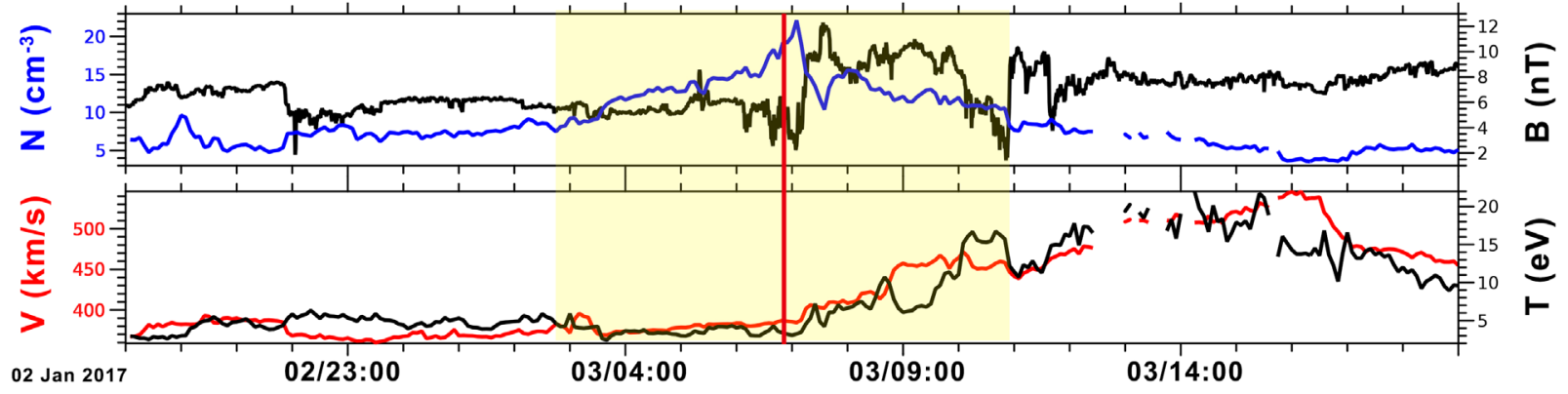

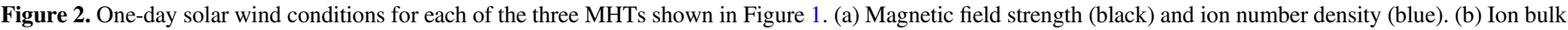

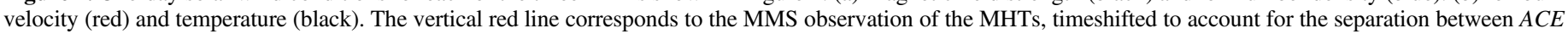
and MMS spacecraft, given the observed solar wind velocity. Panels (c)-(d) and (e)-(f) are in the same format as panels (a)-(b).

detected near the subsolar point in front of the bow shock, which can be regarded as the Earth's foreshock region. From Figures 1(a)-(c), one can find several repeating structures in which the magnetic field rapidly decreased and recovered, and appeared to be in a continuous train-like pattern. The depths of MHTs 1 and 2 (the difference value between the magnetic strength at the edge and the minimum value inside a hole) range from several $\mathrm{nT}$ to more than $10 \mathrm{nT}$, while the depths of MHT 3 range in $0.5 \sim 1 \mathrm{nT}$. The spatial scales of these MHs were close to or less than $\rho_{\mathrm{i}}$, and temporal scales were less than $0.5 \mathrm{~s}$.

Figure 2 gives the detailed solar wind conditions provided by the $A C E$ spacecraft over a one-day interval for each event. The solar wind conditions corresponding with the MHTs are marked by red vertical lines in the plots, applying a time shift in each case to account for the separation between $A C E$ and $M M S$ spacecraft ( $\sim 200$ Earth radii) using solar wind velocities of $390 \mathrm{~km} \mathrm{~s}^{-1}, 330 \mathrm{~km} \mathrm{~s}^{-1}$, and $380 \mathrm{~km} \mathrm{~s}^{-1}$ for MHTs 1,2 , and 3 , respectively. It is clear that all three events occurred during strong ion density enhancements, increasing from a typical value of $\sim 5 \mathrm{~cm}^{-3}$ to a large value up to $\sim 20 \mathrm{~cm}^{-3}$ and lasting for $5-8 \mathrm{hr}$, indicated by the yellow highlighted intervals in each plot. The density enhancements were followed by faster solar wind velocities in all three MHTs. These characteristics suggest that the conditions in which MHTs were observed are likely to correspond with a compression region, where high-speed solar wind flow catches up to preceding low-speed solar wind flow, i.e., a corotating interaction region (CIR) or similar structure in interplanetary space. Therefore, it is reasonable to conclude that these MHTs were observed in the foreshock and its downstream turbulence when a CIR interacted with the Earth's magnetosphere. Since these three MHTs have very similar characteristics, we take MHT 1 as a typical event and present its details in Figures 3-4.

Figure 3 plots details of plasma observations for MHT 1. Figures 3(a)-(b) display the magnetic field strength and components of five possible KSMHs (marked by the vertical red lines), and shows that the magnetic field strength was most strongly decreased in the $z$ component. The electron number density (Figure 3(c)) shows a clear anticorrelation with the magnetic field strength. Figures $3(\mathrm{~d})$ and (e) show the perpendicular and parallel ion and electron temperatures, 


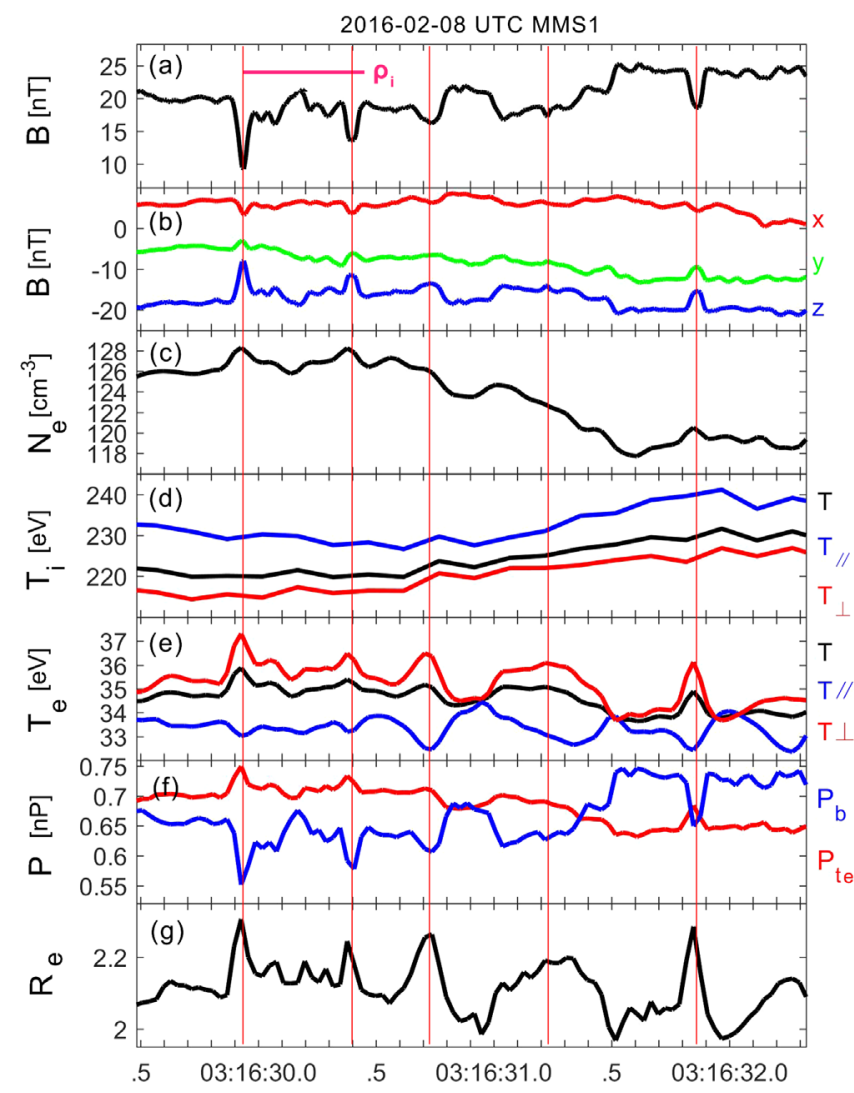

Figure 3. Detailed MMS 1 observations of MHT 1. ((a)-(b)) Magnetic field strength and components. (c) Electron number density. ((d)-(e)) Ion and electron temperature. (f) Magnetic and thermal pressure. The magnetic pressure is shown as increasing by $0.5 \mathrm{nP}$. (g) Electron mirror-mode instability condition.

respectively, and show that although the ion temperature is greater than that of the electrons, there are no appreciable changes in ion temperature with the MHT structure (this is in line with expectations for structures with scale size smaller than the ion gyroradius; e.g., Eastwood et al. 2016; Yao et al. 2017). In contrast, the perpendicular electron temperature is significantly increased inside the KSMHs, while the parallel electron temperature is reduced. In Figure 3(f), the thermal pressure shows a clear anticorrelation with magnetic pressure. Figure $3(\mathrm{~g})$ shows the electron mirror instability condition $R_{\mathrm{e}}=\frac{T_{\mathrm{e} \perp} / T_{\mathrm{e} \|}}{1+1 / \beta_{\mathrm{e} \perp}}$, and has a value clearly greater than 1 during the whole interval, indicating the electron mirror instability threshold is satisfied. Figures 4(b)-(e) display the electron pitch angle distributions from 78 to $1581 \mathrm{eV}$. The loss cone angle $\theta$ for a magnetic mirror, given by $\sin \theta=\sqrt{B / B_{\max }}$, where $B$ and $B_{\max }$ are the local and maximum magnetic field strength, is overplotted (solid black lines) in each figure. The angle $\theta$ is the critical angle for distinguishing between untrapped and trapped particles in the mirror structure. It is clear that the critical trapped angle matches well with abrupt changes in the electron pitch angle distributions. Another very important feature of the mirror mode is its zero-frequency feature, indicating nonpropagating in the plasma flow frame. The MMS four-satellite project has the capability of determining the three-dimensional velocity and direction of structures in space. However, the scale of the KSMH is very small, which makes it difficult for the four satellites to observe similar configurations. In previous MMS observation studies, there were no clear KSMH that were observed by all four spacecraft. Fortunately, the second KSMH in MHT 1 (marked by the second red vertical line in Figures 3(a)-(g)) was observed by all four MMS spacecraft with clear separations and similar configurations during 03:16:30.35 03:16:30.50 UT, as shown in Figure 4(f). This situation is suitable to use the timing method (Russell et al. 1983; Paschmann et al. 1998) to obtain the propagation velocity and the corresponding normal direction. The associated uncertainties were calculated by computing the cross-correlation functions of each spacecraft pair; a detailed description can be found in Knetter (2005), Yao et al. (2018a), and Shi et al. (2019). Here we apply a similar method to this KSMH. In total 5324 times of timing calculation results were carried out, based on the data marked by the light gray shadow in Figure 4(f). The results are displayed as distributions in Figures 4(g)-(h). The distribution standard deviations indicate the uncertainty of the results. The propagation velocity in the ion flow frame is calculated as $V_{\mathrm{P}}=U-\boldsymbol{V} \cdot \boldsymbol{n}$, where $\boldsymbol{V}$ is the averaged background ion flow, $V_{\mathrm{P}}$ and $U$ are the propagation velocity in ion plasma flow frame and spacecraft frame, respectively, and $\boldsymbol{n}$ is the normal direction of $U$. The result indicates that the structure was nonpropagating $\left(4.2 \pm 8.6 \mathrm{~km} \mathrm{~s}^{-1}\right.$, which is indistinguishable from $0 \mathrm{~km} \mathrm{~s}^{-1}$ given the observational uncertainty) in the plasma flow frame.

\section{Summary and Discussion}

To summarize, these MH structures: (1) show train-like features similar to the MHD-scale mirror mode; (2) show anticorrelation between magnetic strength and electron pressure; (3) satisfy the electron mirror-mode excitation condition; (4) have pitch angle distributions that exhibit clearly distinct trapped and untrapped particle populations; (5) are nonpropagating in the plasma flow frame. These observational features are direct evidence of the electron mirror mode. The electron anisotropy is very important and is related to several significant physical processes (e.g., plasma waves and instability) and unsolved problems (e.g., energy conversion and dissipation in turbulence and the heating and acceleration of coronal electrons). The electron mirror mode is one of the important embodiments of electron anisotropy. Historically, the electron mirror mode was studied in theories and numerical simulation in previous studies, and the existence of such structures has remained a subject of debate. Using unprecedented high temporal resolution MMS data, we provide clear observational evidence for the existence of electron mirror modes in space.

In previous particle-in-cell simulations (Hellinger \& Štverák 2018), electron mirror modes consisted of train-like structures and were essentially nonpropagating with weak anticorrelation between electron number density and magnetic field strength. These features agree well with our observations. Noreen et al. (2017) discussed the contribution of electrons in the quasilinear regime of the mirror instability. Their study shows that although the linear growth rate of the electron mirror mode can be much higher than that of the proton mirror mode, the dynamic importance of the electron mirror mode is insignificant since their influence on magnetic field and particle temperature is negligible. Treumann \& Baumjohann (2018) suggested that the quasilinear theory as a saturation mechanism does not apply to the mirror mode in space since the observed amplitudes exceed those predicted by quasilinear theory. Their study using old spacecraft data is motivational for future investigations using the high temporal and spatial resolution data set of the MMS mission. In our study, the MMS magnetic 


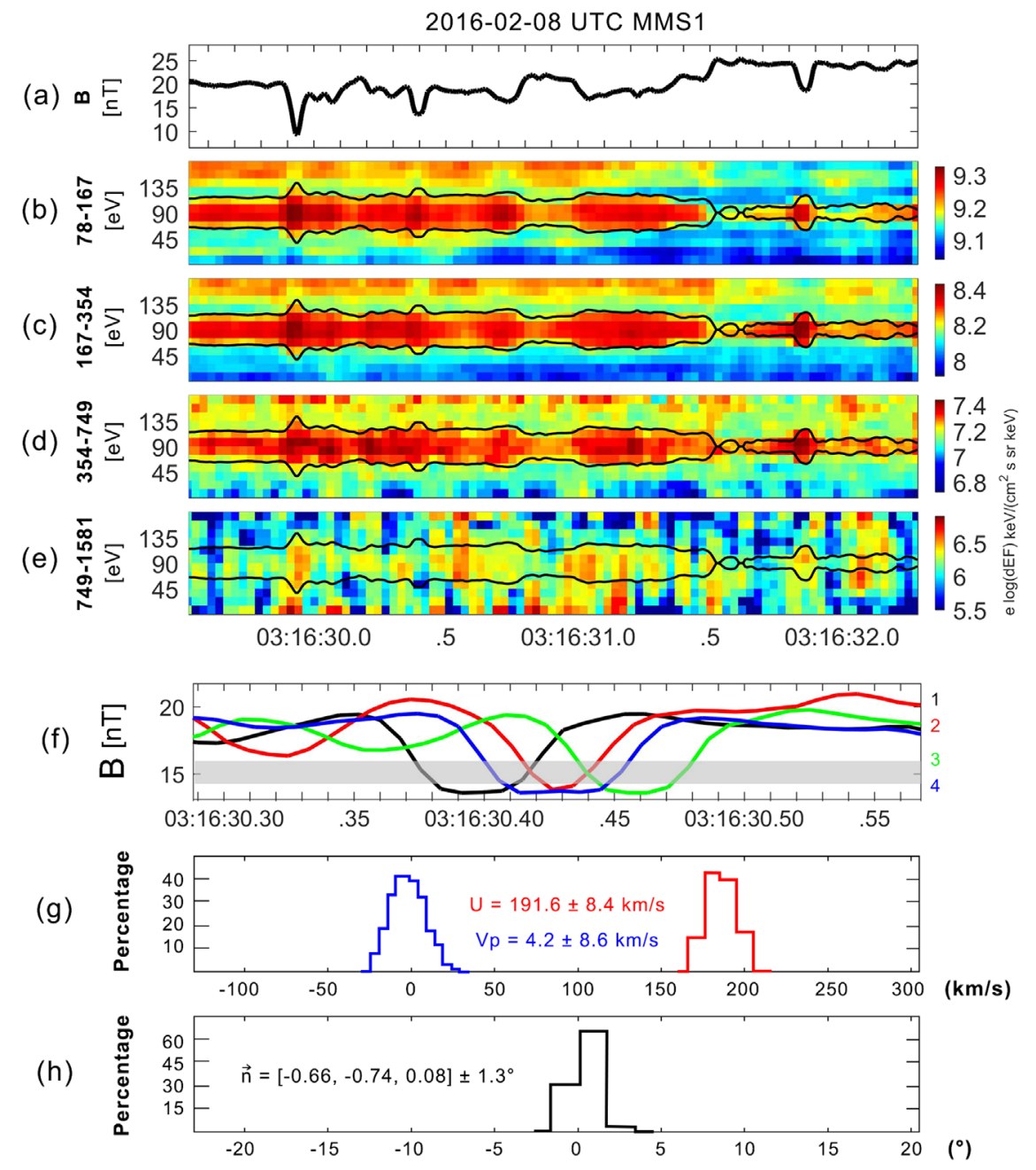

Figure 4. (a) Magnetic field strength. ((b)-(e)) Electron pitch angle distributions. The black lines are the mirror-mode critical trapped angle. (f) Magnetic field strength of MMS 1-4. The data samples within the light gray shadow are used to calculate the timing velocity. $((\mathrm{g})-(\mathrm{h}))$ Histograms of the timing results of the velocity and normal direction. $V_{\mathrm{P}}$ and $U$ are the propagation velocity in the ion plasma flow frame and spacecraft frame, and $\boldsymbol{n}$ is the normal direction of $U$.

field data show that the amplitudes of the electron mirror mode are significant. For example the amplitudes of MHT 3 are greater than $10 \%$ and are greater than $50 \%$ of MHT 1 and MHT 2. A very interesting question is whether or not the electrons play important roles in the mirror-mode physics. Previous studies claimed that electrons have very little or auxiliary effects on the mirror mode. However, when considering the large-scale differences between electrons and ions, although the electrons are much colder than the ions (Figures 3(d)-(e)), the electron mirror mode can be excited with a large amplitude and a scale size less than an ion gyroradius. Under such conditions ions may be treated as a background. Our observation shows that the mirror mode excited by electrons can be intensively excited, indicating their important roles in kinetic-scale plasma physics. Recent studies also show the importance of electrons in the evolution even in the ion mirror mode (e.g., Shaaban et al. 2018; Treumann \& Baumjohann 2019). The electron mirror-mode structures were observed in the foreshock and its downstream regions, which are highly turbulent, and rich in particle acceleration, energy conversion, and mass and momentum transport. The interaction between the CIR and Earth's magnetosphere increases the plasma beta and causes perturbations on different scales. These electron mirror-mode structures could participate in the plasma turbulence cascade from ion to electron scales and could be involved with the energy transfer from the CIR to Earth's magnetosphere. These observations provide an observational basis for a better understanding of mirror mode and turbulence in space, astrophysical, and laboratory plasmas and should also promote the development and improvement of theories and numerical simulation.

We acknowledge the use of the magnetic field and plasma data of ACE from the CDPP-AMDA team and the MMS instrumental teams. All the data are available from MMS Science Data Center (https://lasp.colorado.edu/mms/sdc/ public/). This work was supported by the National Natural Science Foundation of China (grants 41774153, 41574157, 41674165 , and 41431072), project supported by the Specialized Research Fund for State Key Laboratories, the International Space Science Institute (ISSI). H.Z. is partially supported by NSF AGS-1352669. Z.H.Y. is supported by the PRODEX program managed by ESA in collaboration with the Belgian Federal Science Policy Office. 


\section{ORCID iDs}

S. T. Yao ๑ https://orcid.org/0000-0002-6059-2963

Q. Q. Shi (i) https://orcid.org/0000-0001-6835-4751

Z. H. Yao (1) https://orcid.org/0000-0001-6826-2486

R. L. Guo ๑i https://orcid.org/0000-0002-7125-0942

I. J. Rae (i) https://orcid.org/0000-0002-2637-4786

C. T. Russell (i) https://orcid.org/0000-0003-1639-8298

\section{References}

Ahmadi, N., Wilder, F. D., Ergun, R. E., et al. 2018, JGRA, 123, 6383 Balikhin, M. A., Sibeck, D. G., Runov, A., \& Walker, S. N. 2012, JGRA, 117, A08229

Basu, B., \& Coppi, B. 1982, PhRvL, 48, 799

Basu, B., \& Coppi, B. 1984, PhFl, 27, 1187

Baumgärtel, K. 1999, JGR, 104, 28,295

Breuillard, H., Le Contel, O., Chust, T., et al. 2018, JGRA, 123, 93

Burch, J. L., Moore, T. E., Torbert, R. B., \& Giles, B. L. 2016, SSRv, 199, 5

Chisham, G., Burgess, D., Schwartz, S. J., \& Dunlop, M. W. 1998, JGR, 103 , 26,765

Eastwood, J. P., Phan, T. D., Cassak, P. A., et al. 2016, GeoRL, 43, 4716

Gary, S. P., \& Karimabadi, H. 2006, JGRA, 111, A11224

Genot, V., Budnik, E., Hellinger, P., et al. 2009, AnGeo, 27, 601

Gershman, D. J., Dorelli, J. C., Viñas, A. F., et al. 2016, GeoRL, 43, 4112

Goodrich, K. A., Ergun, R. E., Wilder, F. D., et al. 2016, GeoRL, 43, 5953

Hasegawa, A. 1969, PhFl, 12, 2642

Hellinger, P., Kuznetsov, E. A., Passot, T., Sulem, P. L., \& Trávníèek, P. M. 2009, GeoRL, 36, L06103

Hellinger, P., \& Štverák, Š. 2018, JPIPh, 84, 905840402

Huang, S. Y., Sahraoui, F., Yuan, Z. G., et al. 2017, ApJL, 836, L27

Kivelson, M. G., \& Southwood, D. J. 1996, JGR, 101, 17,365

Knetter, T. 2005, A new perspective of the solar wind microstructure due to multi-point observations of discontinuities, $\mathrm{PhD}$ thesis, Univ. zu Köln

Li, Z.-Y., Sun, W.-J., Wang, X.-G., et al. 2016, JGRA, 121, 4180

Liu, H., Zong, Q.-G., Zhang, H., et al. 2019, NatCo, 10, 1040

Lucek, E. A., Dunlop, M. W., Balogh, A., et al. 1999, GeoRL, 26, 2159
Marchenko, V. A., Nezlina, Y. M., \& Pokhotelov, O. A. 1988, PPCF, 30, 957 Migliuolo, S. 1986, JGR, 91, 7981

Noreen, N., Yoon, P. H., López, R. A., \& Zaheer, S. 2017, JGRA, 122, 6978

Paschmann, G., Fazakerley, A. N., \& Schwartz, S. J. 1998, Moments of Plasma Velocity Distributions. In Analysis Methods for Multi-spacecraft Data (Noordwijk: ISSI SA Publications Division), 125

Plaschke, F., Karlsson, T., Götz, C., et al. 2018, A\&A, 618, A114

Pokhotelov, O. A., Balikhin, M. A., Alleyne, S. C. K., \& Onishchenko, O. G. 2000, JGR, 105, 2393

Pokhotelov, O. A., Onishchenko, O. G., \& Stenflo, L. 2013, PhyS, 87, 065303

Pokhotelov, O. A., Sandberg, I., Sagdeev, R. Z., et al. 2003, JGRA, 108, 1098 Pollock, C., Moore, T., Jacques, A., et al. 2016, SSRv, 199, 331

Russell, C. T., Anderson, B. J., Baumjohann, W., et al. 2016, SSRv, 199, 189

Russell, C. T., Jian, L. K., Luhmann, J. G., et al. 2008, GeoRL, 35, L15101

Russell, C. T., Mellott, M. M., Smith, E. J., \& King, J. H. 1983, JGR, 88, 4739

Russell, C. T., Riedler, W., Schwingenshuh, K., \& Yeroshenko, Y. 1987, GeoRL, 14, 644

Shaaban, S. M., Lazar, M., Astfalk, P., \& Poedts, S. 2018, JGRA, 123, 1754

Shi, Q. Q., Pu, Z. Y., Soucek, J., et al. 2009, JGRA, 114, A10202

Shi, Q. Q., Tian, A. M., Bai, S. C., et al. 2019, SSRv, 215, 35

Smith, E. J., Holtzer, R. E., \& Russell, C. T. 1969, JGR, 74, 3027

Southwood, D. J., \& Kivelson, M. G. 1993, JGR, 98, 9181

Stasiewicz, K. 2004, PhRvL, 93, 125004

Sun, W. J., Shi, Q. Q., Fu, S. Y., et al. 2012, AnGeo, 30, 583

Sundberg, T., Burgess, D., \& Haynes, C. T. 2015, JGRA, 120, 2600

Treumann, R. A., \& Baumjohann, W. 2018, AnGeo, 36, 1563

Treumann, R. A., \& Baumjohann, W. 2019, Ann. Geophys. Discuss., in press, doi:10.5194/angeo-2019-86

Winterhalter, D., Neugebauer, M., Goldstein, B. E., et al. 1994, JGR, 99, 23,371

Xiao, T., Shi, Q. Q., Tian, A. M., et al. 2014, SoPh, 289, 3175

Yao, S. T., Shi, Q. Q., Guo, R. L., et al. 2018a, GeoRL, 45, 527

Yao, S. T., Shi, Q. Q., Li, Z. Y., et al. 2016, JGRA, 121, 5510

Yao, S. T., Shi, Q. Q., Liu, J., et al. 2018b, JGRA, 123, 5561

Yao, S. T., Shi, Q. Q., Yao, Z. H., et al. 2019, GeoRL, 46, 523

Yao, S. T., Wang, X. G., Shi, Q. Q., et al. 2017, JGRA, 122, 1999

Zhang, X.-J., Artemyev, A., Angelopoulos, V., \& Horne, R. B. 2017, JGRA, $122,10,304$ 